\title{
The effect of light quality on the expression of stomatal development genes of maize
}

\author{
T.D. LIU ${ }^{*, * * *, \#, ~ X . W . ~ Z H A N G ~}{ }^{* *, * * *}$, and Y. XU $U^{* *, * * *,+}$ \\ College of Crop Sciences, Fujian Agriculture and Forestry University, Fujian, 350002 Fuzhou, China* \\ College of Mechanical and Electronic Engineering, Fujian Agriculture and Forestry University, Fujian, \\ 350002 Fuzhou, China** \\ Center of Excellence for Research in Optoelectronic Agriculture, Fujian Agriculture and Forestry University, \\ Fujian, 350002 Fuzhou, China ${ }^{* * *}$ \\ Key Laboratory of Ministry of Education for Genetics, Breeding and Multiple Utilization of Crops, College of Crop \\ Science, Fujian Agriculture and Forestry University, Fujian, 350002 Fuzhou, China
}

\begin{abstract}
Light-induced stomatal development is mediated through a crosstalk between cryptochrome (CRY), phytochrome (phy), and constitutive photomorphogenic 1 (COP1)-signaling systems. In maize, crosstalk is a complicated phenomenon that is accomplished by COP1-signaling pathway. Epidermal patterning factor 2 (EPF2) and epidermal patterning factor-like 9 (EPFL9)-regulated path signals rely on CRY and phy under blue and red light, respectively. Our experimental results showed that, in maize, ZmCOP1 expressed downstream CRY under monochromatic blue light. Blue light, which induced $Z m E P F 2$ expression, was restricted, thereby increasing stomatal formation through the increase in stomatal index and density. Moreover, blue light reduced the expression of $\mathrm{ZmSPCH}$ and ZmMUTE. By contrast, red light promoted the expression of ZmEPF2 and ZmEPFL9, and mainly acted as a negative signal for regulation. As a consequence, the activatorinhibitor system of light was induced by blue-and-red light cross-effect on the EPF/EPFL peptides. The mediating action of monochromatic light was reduced under polychromatic light.
\end{abstract}

Additional key words: gas-exchange parameters; plant morphology; stomatal density; stomatal index.

\section{Introduction}

The ability of stomata to regulate gas exchange in and out of leaves allows plants to adapt to various types of environments and affects global water and carbon cycles (Hetherington and Woodward 2003). The number of stomata, which developed in leaf epidermis, is affected by several environmental variables, including lighting conditions and atmospheric $\mathrm{CO}_{2}$ concentrations. The understanding on the network of interacting signals, which regulate stomatal differentiation, has improved (Le et al. 2014). However, the influence of such environmental factors on stomatal patterning is still poorly understood. Plant's photoreceptor systems have evolved multiple light responses, such as response to light quality, quantity, and direction. Important regulating photoreceptors include blue light photoreceptors, cryptochromes (CRYs) (Lin 2002), and red/far-red light photoreceptors, phytochromes (phy). Light signals received by CRYs and phytochromes mediate photomorphogenic development, photoperiodic flowering (Casal and Mazzella 1998), stomatal movement (Mao et al. 2005), and stomatal development (Casson et al. 2009).

Both red and blue lights control stomatal development, and their respective independent regulatory pathways are also generally clear. Blue light suppresses the expression of CRYs (Lin 2002) and directly regulates stomatal formation by controlling constitutive photomorphogenic 1(COP1) and CRY mutants with CRYs. Nevertheless, the regulatory function of red light is recognized by photoreceptor phytochrome $\mathrm{B}$ (phyB), which plays a role both within the stomatal lineage and nonepidermal tissues to regulate the changes of the cell fate during stomatal development. SPCH, MUTE, and FAMA are downstream transcription factors that directly control the stomatal development and are all decreased during the downregulation of phyB (Casson et al. 2014). Stomatal formation is inhibited even with the overexpression of $\mathrm{SPCH}$, MUTE, and FAMA. Although its mechanism is poorly

Received 4 June 2018, accepted 21 November 2018.

${ }^{+}$Corresponding author; phone: +86 5918375 6227, e-mail: y.xu@,fafu.edu.cn

Abbreviations: B - blue light; $\mathrm{BR}$ - blue light + red light; $C_{\mathrm{i}}$ - intercellular $\mathrm{CO}_{2}$ concentration; $\mathrm{COP} 1$ - constitutive photomorphogenic 1; $\mathrm{CRY}$ - cryptochrome; $E$ - evapotranspiration; EPF - epidermal patterning factor; EPFL - epidermal patterning factor-like; $g_{\mathrm{s}}-$ stomatal conductance; LMA - leaf mass per area; P - purple light; phy - phytochrome; $P_{\mathrm{N}}$ - net photosynthetic rate; PR - purple + red light; $\mathrm{R}$ - red light; SD - stomatal density; SI - stomatal index.

Acknowledgements: This research was supported by the Major Scientific Project of Fujian Province, China (Grant No. 2014NZ0002-2) and Scientific and Technology Project of Education Department of Fujian Province, China, (No. JAT160164), and Key Project of Fujian Province, Breeding and Industrialization of Major Vegetable with High Yield, Improved Quality, Resistance and Wide Adaptability (2018NZ0002-2). 
understood, the role of red light as a regulatory signal is remarkably noticeable. Light-controlled stomatal development is possibly mediated through a crosstalk within CRY-phytochrome-COP1 signaling system. COP1 acts as a genetically downstream of CRY, phyA, and phyB, and in parallel with the leucine-rich repeat receptorlike protein too many mouths (TMM) but upstream of YDA, SPCH, MUTE, and FAMA (Kang et al. 2009). COP1 is the key negative regulator acting constitutively on repressing stomatal development and differentiation in both light and dark conditions (Wang et al. 2001). However, the regulatory effect of COP1 on environmental factors, especially during light period, is rarely reported. Stomatal development is positively regulated by EPFL9, and its expression in the mesophyll is regulated by light (Hronková et al. 2015). Epidermal patterning factor (EPF) and EPF-like (EPFL) peptides from the same family can produce opposite effects on stomatal density (Ohki et al. 2011). However, the functional competition observed in TMM was not caused by a direct interaction between EPFL9 and epidermal patterning factor 2 (EPF2) (Kondo et al. 2010). Independent red light considerably increases stomatal protein expression compared to blue or purple light. However, our previous experimental results revealed that the simultaneously high expression of EPF2 and EPFL9 is not enough for stomatal development (Liu et al. 2017). The negative regulation of other factors has greatly disturbed this process.

Maize is a typical $\mathrm{C}_{4}$ plant; its biochemical process is highly sensitive to light conditions. We hypothesize that the two following phenomena occur during stomatal development in maize. First, the regulatory effects of red and blue light interact, and the regulatory effect of red light may be affected by blue light or other regulatory factors, and vice versa. Second, downstream genes, are regulated by EPF2 and EPFL9 in a complex manner. This process may affect the final expression of downstream genes and the true pattern of stomatal development. In order to verify the two above assumptions, we set up an experiment that includes six different light treatments, namely, purple, purple + red, blue, blue + red, red, and white, for genetic analysis and stomatal patterning.

\section{Materials and methods}

Plant materials and growth conditions: Maize genotype Xianyu 335 was cultivated in an LED growth chamber for $10 \mathrm{~d}$. The plants were subjected to the following six light treatments: purple light with wavelength of $425 \mathrm{~nm}(\mathrm{P})$, purple light with wavelength of $425 \mathrm{~nm}+$ red light with wavelength of $660 \mathrm{~nm}$ (PR), blue light with wavelength of $450 \mathrm{~nm}$ (B), blue light with wavelength of $450 \mathrm{~nm}+$ red light with wavelength of $660 \mathrm{~nm}(\mathrm{BR})$, red light with wavelength of $660 \mathrm{~nm}(\mathrm{R})$, and fluorescent white light as the control. Each light treatment was repeated thrice. Light in-tensity of each treatment was set to $150 \mu \mathrm{mol}($ photon) $\mathrm{m}^{-2} \mathrm{~s}^{-1}$. If the light was composed of two different colors, then the ratio of each color was 1:1. Cultivated maize plants were constantly exposed to light environment without a dark period. All maize seedlings were cultivated with hydro-ponic nutrient solution of Japanese garden formula (1966). Cultivating temperature and moisture level were set at $25^{\circ} \mathrm{C}$ and $70 \%$, respectively.

Determination of stomatal index, density, photosynthetic function, and leaf morphology: Five seedlings of each treatment were selected to calculate stomatal density (SD) and stomatal index (SI). Two frames of $0.84 \mathrm{~mm}^{2}$ were counted per leaf of each seedling in the middle of the fourth leaf blade. SD was calculated in terms of the num-ber of stomata cells per $\mathrm{mm}^{2}$ in a square area of 0.84 $\mathrm{mm}^{2}$. SI was calculated using the equation $\mathrm{SI}=$ number of stomata/(number of stomata + number of pavement cells) $\times 100 \%$. Stomata and pavement cells were counted using a Leica DM2500 microscope (Leica Microsystems Inc., Germany); only stomata with pores were counted.

The fourth leaf of maize was selected to measure photosynthetic parameters, including net photosynthesis $\left(P_{\mathrm{N}}\right)$, intercellular $\mathrm{CO}_{2}$ concentration $\left(C_{\mathrm{i}}\right)$, stomatal conductance $\left(g_{\mathrm{s}}\right)$, and transpiration rate $(E)$, by a Li6400 portable photosynthesis system (Licor Biosciences, USA) with $02 B$ red-blue LED leaf chamber, where light intensity was set at $1,200 \mu \mathrm{mol}$ (photon) $\mathrm{m}^{-2} \mathrm{~s}^{-1}$. The measuring position was selected at the middle of the leaf blade. The measurement was repeated on five different plants. Measuring temperature and moisture were set at $25^{\circ} \mathrm{C}$ and $70 \%$, respectively. Plant height, whole plant dry mass, leaf area, and leaf mass per area (LMA) were measured. The dry mass of leaf and whole plant were measured after $12 \mathrm{~h}$ in drying box at $85^{\circ} \mathrm{C}$.

RNA extraction and quantitative Real-Time PCR (qRT-PCR): The upper part of the fourth leaf with five replicas was selected for PCR. Total RNA was isolated using the RNAqueous ${ }^{\circledR}$ Total RNA Isolation Kit AM1912 (Life Technologies Corp., Grand Island, New York, USA). The RNA yield was determined using a NanoDrop 2000 spectrophotometer (Thermo Scientific, USA), and the integrity was evaluated using agarose gel electrophoresis with ethidium bromide stain. Quantified reactions were performed in a GeneAmp ${ }^{\circledR}$ PCR System 9700 (Applied Biosystems, USA). RT-PCR was performed using LightCycler ${ }^{\circledR} 480$ II Real-time PCR Instrument (Roche, Switzerland) with QuantiFast ${ }^{\circledR} S Y B R \circledR$ Green $q P C R$ Master Mix (Qiagen, Germany). Each sample was repeated three times. The primer sequences were designed in the laboratory and synthesized by Generay Biotech (Generay, China) based on the mRNA sequences obtained from the NCBI database and were as follows:

\begin{tabular}{ll}
\hline Gene name & Primer sequence (5' to 3') \\
\hline ZmCryl & F 5'-GCTTCGGGACACTCTGATTCTT-3' \\
& R 5'-TGCCTGTCCAGATAACCCCTACT-3' \\
ZmPhyB1 & F 5'-CTGGTAGAGAAATCGTCTGATATGTT- \\
& AAG-3' \\
& R 5'-TTCTCGTAAATTAGGCACCCTCTT-3' \\
$Z m C O P 1$ & F 5'-TGGAGGGCGTTCAAGAGATT-3' \\
& R 5'-AATGGCACGAAAGGGAACAC-3' \\
$Z m E P F 2$ & F 5'-TGGTGCCGATCGATTCATTAG-3' \\
& R 5'-CGTAGCAGGTGAGGTCATCATG-3'
\end{tabular}




$\begin{array}{ll}Z m S T O M A \text {-F 5'-TGGAGTCTGGAGGAGCATTT-3' } \\ \text { GEN } & \text { R 5'-GGCTGGCCATTCATACTTTC-3' } \\ Z m M U T E & \text { F 5'-AGTGTTGAGGATCTTGCCTACGA-3' } \\ & \text { R 5'-CTCCTCCTGCGGCTTCTG-3' } \\ \text { ZmFAMA } & \text { F 5'-TCAGGTCACTCATGCCAGGAT-3' } \\ & \text { R 5'-TGCTCTAGCTCTCTTATGAACTCGAT-3' } \\ \text { ZmSPCH } & \text { F 5'-GCAGGCCAAATTCGTTACTATCTAC-3' } \\ & \text { R 5'-GTGGCCTCTGCATCTTGAGATAA-3' } \\ \text { GAPDH } & \text { F 5'-CCACTTCGTCAAGCTCGTCT-3' } \\ & \text { R 5'-AACCCCTACTGGGACTTGAA-3' }\end{array}$

$G A P D H$ was used as a reference gene.

Data analysis: Analysis of variance (ANOVA) was used to analyze the significant differences between the measured data by comparing their means. The significance level was set at $0.05(\alpha)$. Multiple comparisons were used to determine the least significant difference (LSD) at $0.05(\alpha)$ by using SPSS 11.5.

\section{Results}

Stomatal development, functional characteristics, and leaf morphology: Stomatal distribution is shown in Fig. 1, whereas SD and SI are shown in Fig. 2. Maize cultivated in the blue light had significantly higher stomata density than those under the control and R (Fig. 2A). If we consider the effect of the epidermal cells on the leaf area, the probability of stomatal formation under B was also better than that under other light conditions (Fig. 2B). By comparison, monochromatic $\mathrm{R}$ and polychromatic light containing $\mathrm{R}$ induced a low SI. Neither monochromatic

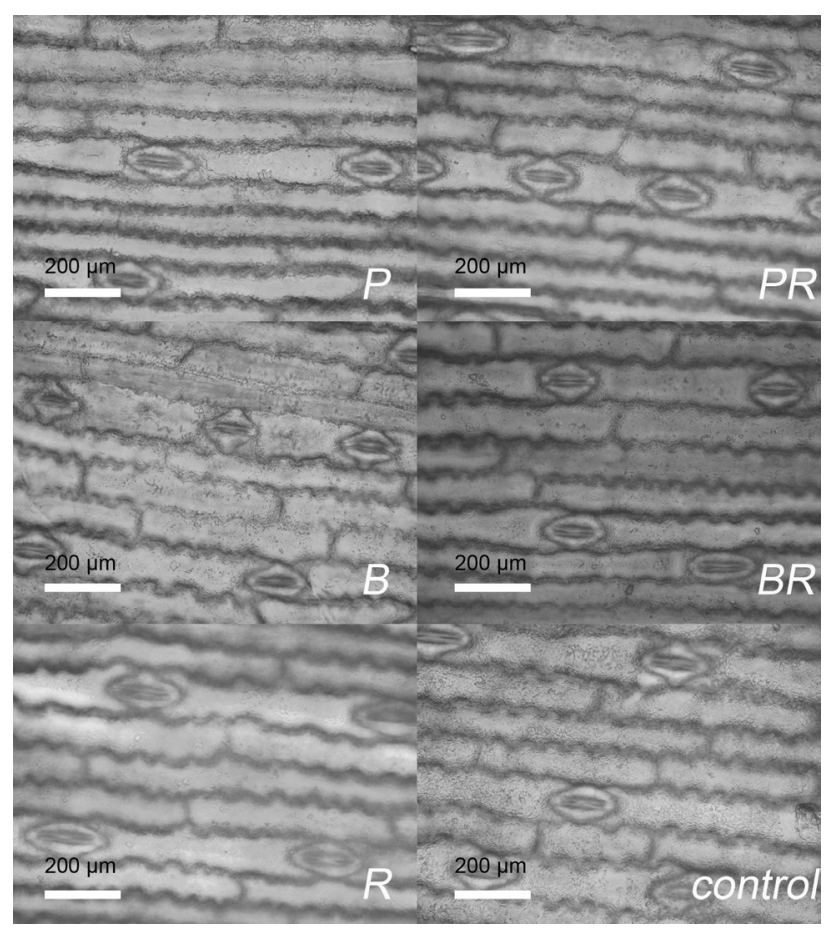

Fig. 1. The distribution of maize seedling's stomata under $400 \times$ microscope under six light conditions $(\mathrm{P}$ - purple, $\mathrm{PR}$ - purple + red, B - blue, BR - blue + red, R - red, and control - white).
$\mathrm{R}$ nor polychromatic light containing $\mathrm{R}$ promoted the stomata formation.

Photosynthesis is an important indicator of stomatal function. Under different environmental stress conditions, the ability of plants to exchange gas was also relatively different. Our experimental results of the $P_{\mathrm{N}}, C_{\mathrm{i}}, g_{\mathrm{s}}$, and $E$ for maize seedlings under six different light conditions are shown in Table 1. Maize cultivated under B and P conditions could significantly promote the $P_{\mathrm{N}}$. Some important parameters determined stomatal function. In this experiment, $g_{\mathrm{s}}$ and $E$ were higher under B and $\mathrm{P}$ than those under the control and R. Although many factors affect photosynthesis and the mechanism of influence is complex, the changes in both $g_{\mathrm{s}}$ and $E$ can still explain that stomatal function may affect photosynthesis. Photosynthetic parameters showed that under B, maize showed high gasexchange ability, which may be caused by the high SD and SI. Moreover, $C_{\mathrm{i}}$ under B was also higher than those under other light conditions.

Leaf morphology depicted in Table 2 showed that the average height of maize seedlings under B was significantly lower than that under other treatments except for control, and their dry mass and leaf area were also lower than that under P. R-promoted maize growth highly expanded the leaf area but accumulated less LMA than other treatments. Dry mass and LMA of PR reached $0.273 \mathrm{~g}$ and $1.35 \mathrm{mg} \mathrm{cm}^{-2}$, respectively, which were the highest values in our experiment. $\mathrm{R}$ promoted excessive growth of maize seedlings, and polychromatic light was beneficial for biomass accumulation.

Light-induced stomatal development mediated by CRY, phytochrome, and COP1: Light quality is one of the most important signals in controlling stomatal development of plants. CRY is the blue light receptor that mediates lightinduced responses in stomatal development. Relative expression of ZmCryl and ZmPhyB1 is shown in Fig. $3 \mathrm{~A}$ and the scattered distributions of SD with $Z m C r y l$ and $Z m P h y B 1$ in Fig. 3B, while SI with ZmCryl and ZmPhyB 1 under six light conditions is shown in Fig. $3 C$. When examining the light response of $Z m C r y 1$ under six light conditions, we observed the dramatically reduced expression of ZmCryl under B. The lack of blue light increased the expression of $\mathrm{ZmCry} 1$, especially in monochrome red light (Fig. 3A). However, the SI and SD under B were remarkably higher than those under R. Other researchers found that Cryl mutant reduced SI considerably than the wild type ones (Kang et al. 2009). Our experiment showed that as a signal of the change in ZmCryl expression, the light quality is more effective than light quantity. Interestingly, $\mathrm{ZmCry} 1$ receptors were strongly reactive to $\mathrm{R}$ and similar to ZmPhyB1.ZmPhyB1 was significantly reduced under $\mathrm{P}$ and $\mathrm{B}$ compared with the white control (Fig. 3A). However, blue was the only light that induced the change of SD and SI conducted by the ZmPhyB 1-signal pathway. Measurement of SD and SI revealed that the low expression levels of ZmCryl and ZmPhyBl increased the SD (Fig. 3B) and SI (Fig. 3C), whereas SD and SI under the BR light treatment were similar to those under the control. The relative expression levels of ZmCryl 


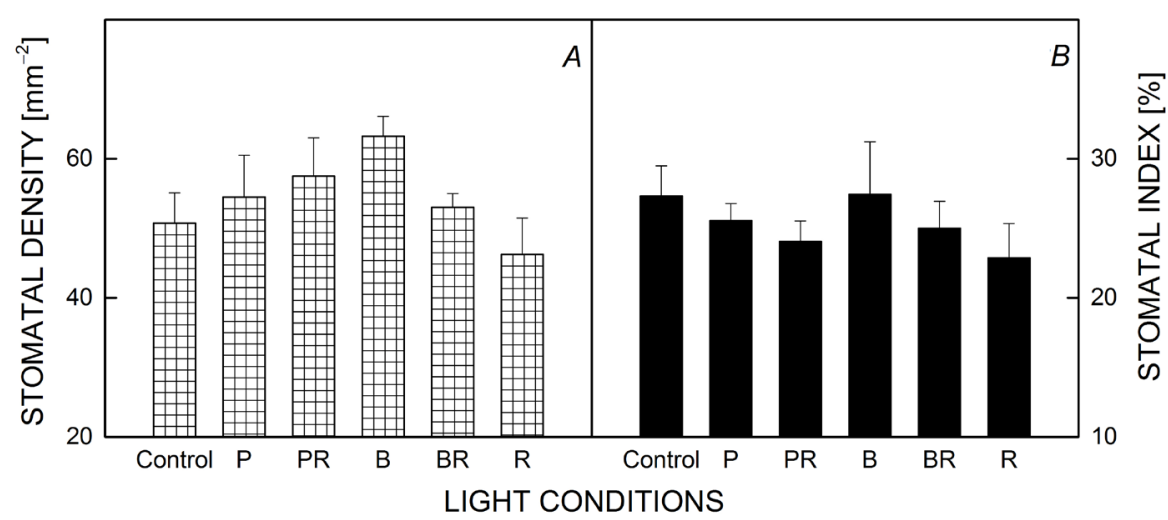

Fig. 2. Stomatal density (SD) $(A)$ and stomatal index $(\mathrm{SI})(B)$ in maize under six light conditions $(\mathrm{P}-$ purple, $\mathrm{PR}-$ purple + red, B - blue, BR - blue + red, $\mathrm{R}$ - red, and control - white). Values are means $\pm \mathrm{SD}, n=10$.

Table 1. Photosynthetic rate $\left(P_{\mathrm{N}}\right)$, intercellular $\mathrm{CO}_{2}$ concentration $\left(C_{\mathrm{i}}\right)$, stomatal conductance $\left(g_{\mathrm{s}}\right)$, and transpiration rate $(E)$ for maize seedlings under six light conditions $(\mathrm{P}-$ purple, $\mathrm{PR}-$ purple + red, $\mathrm{B}-$ blue, $\mathrm{BR}-$ blue + red, $\mathrm{R}-$ red, and control white). The fourth leaf was chosen for measurement on the middle of the leaf. Different letters indicate significant difference (0.05).

\begin{tabular}{lllll}
\hline Light & $P_{\mathrm{N}}\left[\mu \mathrm{mol}\left(\mathrm{CO}_{2}\right) \mathrm{m}^{-2} \mathrm{~s}^{-1}\right]$ & $C_{\mathrm{i}}\left[\mu \mathrm{mol}\left(\mathrm{CO}_{2}\right) \mathrm{mol}^{-1}\right]$ & $g_{\mathrm{s}}\left[\mathrm{mol}\left(\mathrm{H}_{2} \mathrm{O}\right) \mathrm{m}^{-2} \mathrm{~s}^{-1}\right]$ & $E\left[\mathrm{mmol}\left(\mathrm{H}_{2} \mathrm{O}\right) \mathrm{m}^{-2} \mathrm{~s}^{-1}\right]$ \\
\hline $\mathrm{P}$ & $34.03 \pm 1.95^{\mathrm{ab}}$ & $130.5 \pm 10.4^{\mathrm{a}}$ & $0.244 \pm 0.027^{\mathrm{ab}}$ & $5.06 \pm 0.37^{\mathrm{a}}$ \\
$\mathrm{PR}$ & $31.32 \pm 0.66^{\mathrm{b}}$ & $128.6 \pm 9.6^{\mathrm{a}}$ & $0.217 \pm 0.011^{\mathrm{ab}}$ & $4.22 \pm 0.21^{\mathrm{b}}$ \\
$\mathrm{B}$ & $34.39 \pm 1.47^{\mathrm{a}}$ & $134.3 \pm 11.6^{\mathrm{a}}$ & $0.248 \pm 0.024^{\mathrm{a}}$ & $5.02 \pm 0.29^{\mathrm{a}}$ \\
$\mathrm{BR}$ & $31.18 \pm 1.21^{\mathrm{b}}$ & $119.8 \pm 9.8^{\mathrm{a}}$ & $0.210 \pm 0.007^{\mathrm{b}}$ & $3.95 \pm 0.09^{\mathrm{b}}$ \\
$\mathrm{R}$ & $23.30 \pm 1.53^{\mathrm{c}}$ & $124.4 \pm 18.4^{\mathrm{a}}$ & $0.149 \pm 0.022^{\mathrm{c}}$ & $3.42 \pm 0.39^{\mathrm{c}}$ \\
control & $32.03 \pm 1.04^{\mathrm{ab}}$ & $132.4 \pm 4.8^{\mathrm{a}}$ & $0.230 \pm 0.012^{\mathrm{ab}}$ & $3.87 \pm 0.25^{\mathrm{bc}}$ \\
\hline
\end{tabular}

Table 2. Experimental results of plant morphology and leaf characteristics of maize seedlings under six light conditions ( $\mathrm{P}-$ purple, $\mathrm{PR}$ - purple + red, B - blue, BR - blue + red, R - red, and control - white). Different letters indicate significant difference (0.05). LMA- leaf mass per area.

\begin{tabular}{lllll}
\hline Light & Height $[\mathrm{cm}]$ & Dry mass $[\mathrm{g}]$ & Leaf area $\left[\mathrm{cm}^{2}\right]$ & LMA $\left[\mathrm{mg} \mathrm{cm}^{-2}\right]$ \\
\hline P & $29.42 \pm 0.33^{\mathrm{b}}$ & $0.247 \pm 0.023^{\mathrm{a}}$ & $50.69 \pm 1.45^{\mathrm{b}}$ & $1.31 \pm 0.03^{\mathrm{a}}$ \\
PR & $29.66 \pm 0.75^{\mathrm{b}}$ & $0.273 \pm 0.039^{\mathrm{a}}$ & $54.82 \pm 2.19^{\mathrm{a}}$ & $1.35 \pm 0.03^{\mathrm{a}}$ \\
$\mathrm{B}$ & $24.06 \pm 0.83^{\mathrm{c}}$ & $0.242 \pm 0.011^{\mathrm{a}}$ & $47.80 \pm 1.00^{\mathrm{b}}$ & $1.26 \pm 0.01^{\mathrm{b}}$ \\
BR & $24.30 \pm 1.08^{\mathrm{c}}$ & $0.269 \pm 0.021^{\mathrm{a}}$ & $49.96 \pm 1.44^{\mathrm{b}}$ & $1.34 \pm 0.03^{\mathrm{a}}$ \\
R & $34.02 \pm 0.55^{\mathrm{a}}$ & $0.259 \pm 0.024^{\mathrm{a}}$ & $53.83 \pm 2.70^{\mathrm{ab}}$ & $1.23 \pm 0.03^{\mathrm{b}}$ \\
control & $22.14 \pm 0.92^{\mathrm{d}}$ & $0.261 \pm 0.021^{\mathrm{a}}$ & $42.76 \pm 2.39^{\mathrm{c}}$ & $1.35 \pm 0.02^{\mathrm{a}}$ \\
\hline
\end{tabular}
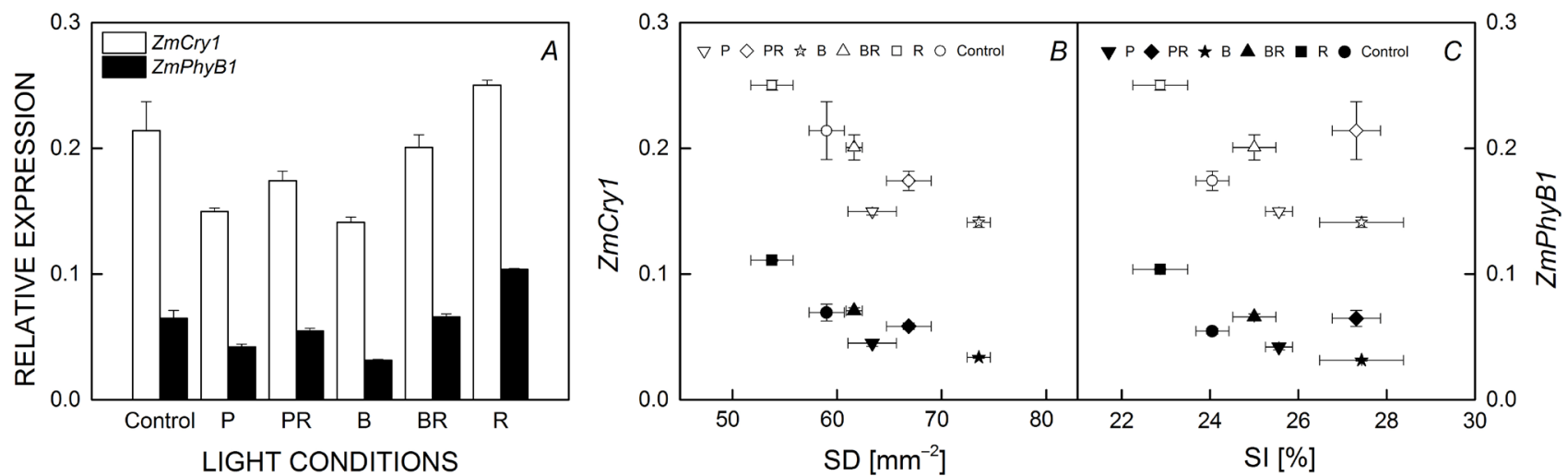

Fig. 3. The relative expression of ZmCryl and $Z m P h y B 1(A)$ and the scattered distributions of stomatal density (SD) with ZmCryl (open) and ZmPhyB1 (solid) (B) and stomatal index (SI) with ZmCryl (open) and ZmPhyB1 (solid) (C) in maize under six light conditions $(\mathrm{P}$ - purple, $\mathrm{PR}$ - purple + red, $\mathrm{B}$ - blue, $\mathrm{BR}$ - blue + red, $\mathrm{R}-$ red, and control - white $)$. Means $\pm \mathrm{SD}, n=3$. 
and $Z m P h y B 1$ under $\mathrm{R}$ were more remarkable than those under the control. However, in the case of SD and SI, the situation was merely the opposite.

$Z m C O P 1$ is the key negative regulator in photomorphogenesis, floral initiation, and stomatal opening (Mao et al. 2005). The signaling mechanism of CRYs is mediated through negative regulation of $\mathrm{ZmCOP} 1$ by direct CRYCOP1 interaction through CRY C-terminal domain. Therefore, in the light-induced signaling pathway, ZmCryl negative regulation results are highly accurate. This finding was also confirmed in this study. Fig. 4 shows the relative expression of $\mathrm{ZmCOP1}$ (Fig. $4 A$ ), and the scattered distribution of SD (Fig. $4 B$ ) and SI (Fig. $4 C$ ) with ZmCOP1 under six light conditions. Although CRY expression was extremely conservative under $\mathrm{B}$, the expression of ZmCOP1 substantially increased (Fig. 4A). In addition, $\mathrm{P}$ remarkably modulated the increase in COP1. ZmCOP1 is regulated by multiple components of light signaling, and the $\mathrm{P}$ - and B-induced negative regulation of $\mathrm{ZmCry} 1$ played a prominent role in this signaling pathway. However, their expression was relatively positively correlated with SD and SI (Fig. 4B). This finding is considerably different from the former report (Kang et al. 2009). Therefore, the role of COP1 under different light conditions still needs further analysis.

Influence of blue light on the expression of $E P F 2$ and the competition of $E P F L 9$ on TMM: The relative expression levels of EPF2 and EPFL9 are the direct controllers that affect the regulated results of upstream gene. The putative secretory peptide EPF2 acts as a negative regulator of stomatal clustering and SD in the early development of leaves. Fig. 5 illustrates the relative expression levels of $Z m E P F 2$ and ZmEPFL9 and the scattered distributions of SD and SI with ZmEPF2 and SD with ZmEPFL9 under six light conditions. Gene expression of ZmEPF2 under B was relatively low, and a high expression was observed under R. As well, SD and SI increased under B compared to R. ZmEPFL9 acted independently of the $Z m E P F 2$ to regulate SD, and the overexpression of it could greatly increase both the SD and SI. Light acted on the expression levels of $Z m E P F 2$ and $Z m E P F L 9$ in different ways. While the expression of ZmEPF2 was the highest under R, white light provided the highest expression of

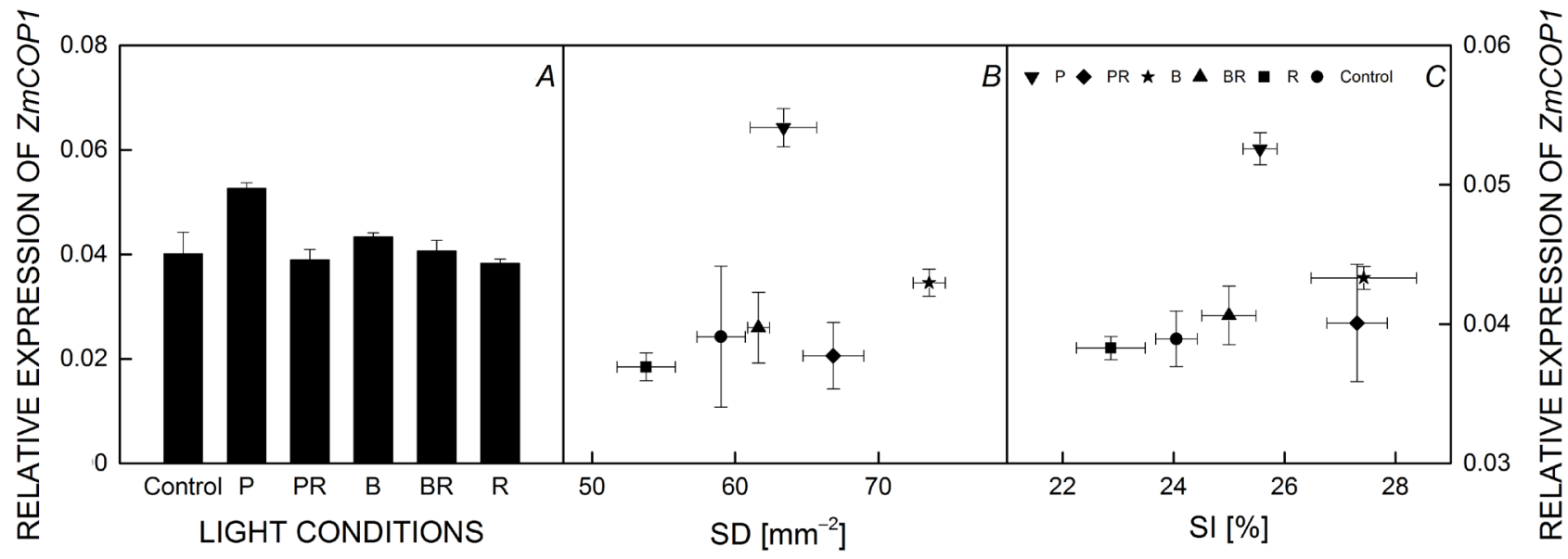

Fig. 4. The relative expression of $Z m C O P 1(A)$ and the scattered distribution of SD $(B)$ and SI $(C)$ with $Z m C O P 1$ in maize under six light conditions $(\mathrm{P}$ - purple, $\mathrm{PR}-$ purple + red, $\mathrm{B}-$ blue, $\mathrm{BR}-$ blue + red, $\mathrm{R}-$ red and control - white). Values are means $\pm \mathrm{SD}, n=3$.
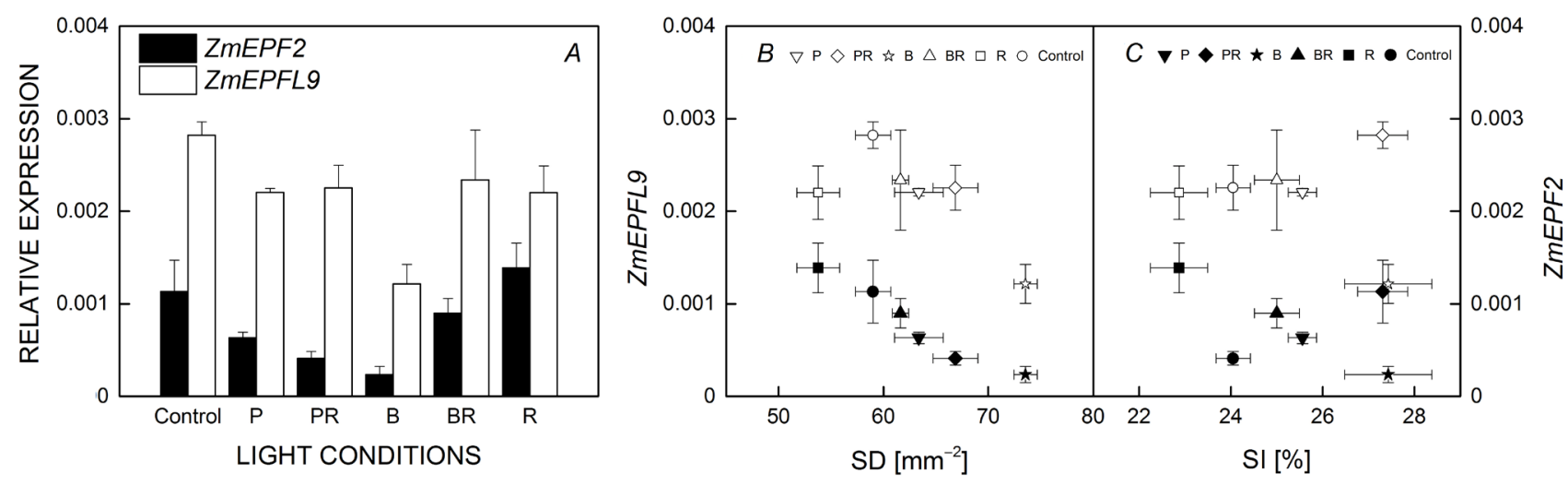

Fig. 5. The relative expression of ZmEPF2 and ZmEPFL9 $(A)$ and the scattered distributions of SD with ZmEPF2 (solid signs) and ZmEPFL9 (open signs) $(B)$ and SI with ZmEPF2 (solid signs) and ZmEPFL9 (open signs) $(C)$ in maize under six light conditions $(\mathrm{P}$ - purple, $\mathrm{PR}-$ purple + red, $\mathrm{B}-$ blue, $\mathrm{BR}-$ blue + red, $\mathrm{R}-$ red and control - white $)$. Values are means $\pm \mathrm{SD}, n=3$. 


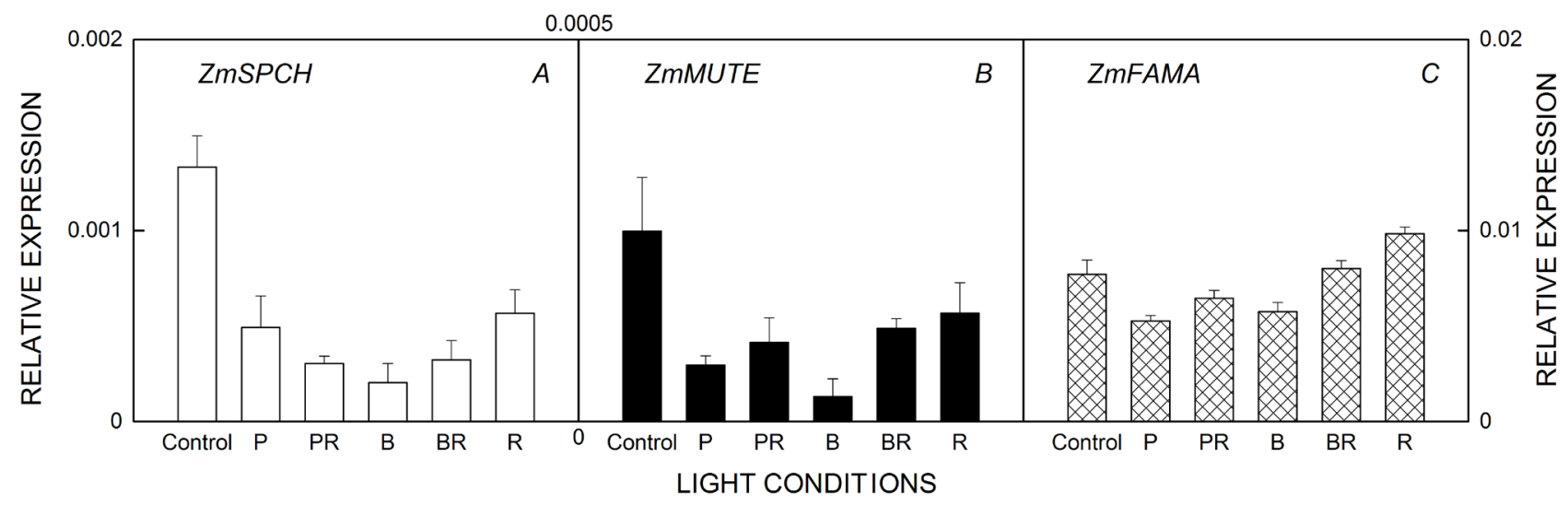

Fig. 6. The relative expression of $\operatorname{ZmSPCH}(A), \operatorname{ZmMUTE}(B)$, and ZmFAMA (C) in maize under six light conditions (P - purple, $\mathrm{PR}-$ purple + red, $\mathrm{B}$ - blue, $\mathrm{BR}-$ blue + red, $\mathrm{R}-$ red and control - white). Values are means $\pm \mathrm{SD}, n=3$.

ZmEPFL9. Nevertheless, B induced the lowest expression of both ZmEPF2 and ZmEPFL9. This phenomenon indicates that the function of negative regulation of $Z m E P F 2$ was more important than that of the positive regulation of EPFL9 in stomatal development. The competitive expression of ZmEPF2 on the TMM greatly inhibited the positive regu-latory effect of ZmEPFL9.

Influence of downstream genes of $S P C H, M U T E$, and $F A M A$ regulated by light on stomatal development: $S P C H, M U T E$, and FAMA genetically act as downstream genes of COP1,EPF2, and EPFL9 to regulate stomatal development and patterning (Le et al. 2014). ZmSPCH, $Z m M U T E$, and ZmFAMA are epistatic to ZmCOP1. The relative expression levels of $Z m S P C H, Z m M U T E$, and ZmFAMA is shown in Fig. 6, while Fig. 1S (supplement) shows the scattered distributions of SD and SI with $\mathrm{ZmSPCH}$ and ZmMUTE under six light conditions. qPCR results demonstrated that the expression levels of ZmSPCH and ZmMUTE were considerably lower under the five LED treatments than that of the control. Among them, the expression levels of ZmSPCH and ZmMUTE under B were significantly lower than that under other light conditions. $Z m C O P 1$ was negatively regulated by CRY and phyB. ZmSPCH, ZmMUTE, and ZmFAMA were in turn negatively regulated by $Z m C O P 1$. Blue light-induced mediation on $\mathrm{ZmSPCH}$ and $\mathrm{ZmMUTE}$ was similar to CRY and $P h y B 1$, thus showing a positive effect on stomatal regulation. The effect of negative regulation reduced the relative expression levels of ZmSPCH, ZmMUTE, and ZmFAMA. From the scattered plot of SI and SD (Fig. 1S), the downregulation of gene expression with blue light increased SD and SI. Moreover, red light upregulated gene expression, which made SI and SD lower. White light enhanced the overexpression of ZmEPFL9 and directly regulated the expression levels of $Z m S P C H$ and $Z m M U T E$; the overexpression of ZmSPCH and ZmMUTE did not promote the improvement of stomatal differentiation.

\section{Discussion}

Blue light-induced stomatal development negatively regulated by EPF2: Zheng and Van Labeke (2017) reported that blue and blue + red light could increase photosynthetic efficiency, SI, and SD. We obtained consistent experimental results. The development of stomata was promoted by blue light, and CRY is involved in this process. Maize seedlings cultivated under monochromatic $\mathrm{B}$ or $\mathrm{P}$ exhibited a decrease in $C R Y 1$, which acts as a regulator that decreases the expression of ZmEPF2 and ZmEPFL9 and increases that of $Z m C O P 1$. COP1 protein acts as an E3 ubiquitin ligase that transduces light signals perceived by photoreceptors. COP1 is a key repressor of light-promoted stomatal development and loss-of-function mutants of COP1 display stomatal clusters, but this phenotype is suppressed by another genetic regulated pathway. Wang (2001) presented that COP1 directly interacted with CRY1 and functioned as a downstream gene of CRYs to regulate the stomatal response to blue light, and the activated CRYs would interact with COP1 to repress its E3 ligase activity, which permits downstream photomorphogenic genes to accumulate and regulate various processes during plant growth and development. Furthermore, light in the blue, red, and far-red wavelength ranges suppresses the COP1mediated degradation of the inducer of CBF expression (ICE) proteins to induce stomatal development (Lee et al. 2017). Our findings revealed that COP1 expression acted as a downstream gene of the CRY under light conditions, especially under monochromatic B. We believe that the effect of COP1 on stomatal patterning under different light conditions might be complicated.

Genetic and biochemical evidence indicates that ERFs react specifically with respect to ligands and developmental stage during stomatal development. The EPF and EPFL genes encode concealed cysteine-rich peptides (Torii 2012). EPF2 is the peptide used as intercellular signals in stomatal patterning. The loss of EPF2 function induces excessive divisions and increases SD. Overexpression of EPF2 represses TMM expression and blocks stomatal formation (Hunt and Gray 2009). In our study, the blue light-induced expression of ZmEPF2 was restricted and consequently increased stomatal formation by increasing SI and SD. Moreover, the blue light-induced low expression of ZmSPCH and ZmMUTE was noteworthy. SPCH, MUTE, 
and FAMA are key regulators that direct three successive stages of stomatal development (Pillitteri et al. 2007) and act as downstream transcriptional factors, which are negatively regulated by COP1. Our experimental analysis of the correlations among CRY, COP1, SPCH, and MUTE were logically coincident. However, the overexpression of $\mathrm{ZmSPCH}$ could increase asymmetric divisions and lead to extra stomata production (Ohashi-Ito and Bergmann 2006). The SPCH level can be decreased by EPF2 but increased by EPFL9 (Jewaria et al. 2013). In our qPCR results, low expression levels of $\mathrm{ZmSPCH}$ and ZmMUTE were detected in blue light. This phenomenon was in line with EPF2 rather than with EPFL9. In addition, SPCH, MUTE, and FAMA may not merely be regulated by light signals and transcriptional level, and posttranscriptional regulation mechanisms might also be involved in this process. The functions of SPCH and MUTE during stomatal initiation require the involvement of some other genetic and biochemical processes (Kanaoka et al. 2008).

Red light restricted stomatal development by positively regulated EPF2 but acted as a promotor to EPFL9: The phyB was involved in the production of stomata that was promoted by red light. The phyB is the primary photoreceptor mediating red light-induced stomatal development. Red light greatly promoted the expression of $Z m P h y B 1$, which acted as a promoting factor to increase the expression of $Z m E P F L 9$. Furthermore, the increased $Z m E P F L 9$ may be regulated by $Z m C O P 1$. Exposure of plants to shade environment increases the nuclear abundance of COP1. In light-grown plants, the activity of COP1 is repressed. In our study, red light played a principal role in this process. As a repressor of photomorphogenesis, which targets many positive regulators of light signaling, red light may serve as one of the reasons for the increment of EPFL9 (Hoecker 2017). EPFL9 is also a member of the EPF/EPFL family of peptides that is concealed from mesophyll cells of immature leaves (Sugano et al. 2010). The lost EPFL9 function using gene silencing via RNA interference resulted in a reduction of the SD (Hunt et al. 2010). EPFL9 increases SD by competing with negative regulators EPF1 and EPF2 for the TMM. In contrast to role of EPF2, the ectopic overexpression of EPFL9 or the application of synthetic EPFL9 peptides induces the formation of clusters containing numerous stomata in the contacting points (Kondo et al. 2010). Relatively different to blue light, red light greatly promoted the expression of $Z m E P F 2$, which mainly functioned as a negative signal except for the regulating path on the EPFL9. However, considering the promotional effect of red light on the EPF2, we found that the positive regulatory effect of EPFL9 has no advantage on the stomatal development in the polychromatic light conditions. EPFL9 expression influenced several implicated genes in the stomatal pathway. However, the EPFL9 may act positively on the SPCH protein level (Lampard and Bergmann 2007) without influencing the transcript level (Jewaria et al. 2013). Nevertheless, EPFL9 expression followed a similar trend with MUTE and FAMA being involved in the specification and differentiation of GC precursors (Lampard and
Bergmann 2007), which is in line with our findings. However, further research is needed on the downstream gene of SPCH, MUTE, and FAMA because their high expression levels did not contribute to the increase in SD and SI. Contrary to previous findings (Hronková et al. 2015), we observed that red light reduced SD, photosynthetic ability, and $\mathrm{CO}_{2}$ uptake accompanied with an elevated ZmEPFL9 expression. This phenomenon would potentially provide a light-induced mechanism for a negative-feedback loop to control the ZmEPFL9 expression under polychromatic light conditions. EPF2 was putatively involved in this loop and consequently affected stomatal development.

Effect of light quality on morphology: R and B effectively drive photosynthesis and promote plant growth (Yang et al. 2017). Although blue light promotes the formation of stomatal development and stomatal function, the development of maize leaf morphological features is relatively stable. Its height and leaf area were slightly higher than that under white light processing. The results of polychromatic light processing are more closely related to the results of the control than those of monochromatic light treatment. LMA was relatively low under control during seedling stage probably due to light quality that regulated other physiological mechanisms. This regulation difference may be prominent in the vegetative growth stage after the seedling stage.

Light-induced stomatal development mediated through a cross-signaling system between the activator and inhibitor: The regulation of light-induced stomatal development demonstrated that the CRY and phyB are the primary photoreceptors mediated by blue and red light, respectively. However, stomatal development is possibly mediated through a crosstalk within the CRYphytochrome-COP1 signaling system. Previous genetic analysis suggested that each of the EPF/EPFL peptides acts independently (Hunt and Gray 2009, Hunt et al. 2010), but the light-induced activator-inhibitor system through the blue and red light was alternately affected on the EPF/ EPFL peptides. As a consequence, the regulative effect of any monochromatic light will be counteracted under the conditions of polychromatic light. For example, in our studies, the increase of $Z m E P F 2$ and $Z m E P F L 9$ under red light was inhibited after adding blue light. Moreover, the expression of $\mathrm{ZmSPCH}, \mathrm{ZmMUTE}$, and ZmFAMA was regulated by several factors under polychromatic lightinduced environment, but their functional mechanism in stomatal development still needs further research. This regulatory network is certainly more complex than our results. Lee et al. (2017) reported that the ICE proteins accumulate in the nuclei of leaf abaxial epidermal cells, which constitutively produce stomata. Light in the blue, red, and far-red wavelength ranges suppresses the COP1mediated degradation of the ICE proteins to induce stomatal development. In addition, SPCH, MUTE, and FAMA constitute a central gene regulatory network along with ICE transcription factors for stomatal functional development. However, their functional mechanism 
in stomatal development still needs further research. Therefore, we concluded that the existence of activators and inhibitors in stomatal development verified that stomatal patterning is controlled by a cross-signaling system and that each factor is not independent of each other.

\section{References}

Casal J.J., Mazzella M.A.: Conditional synergism between cryptochrome 1 and phytochrome $\mathrm{B}$ is shown by the analysis of phyA, phyB, and hy4 simple, double, and triple mutants in Arabidopsis. - Plant Physiol. 118: 19-25, 1998.

Casson S.A., Franklin K.A., Gray J.E. et al.: Phytochrome B and PIF4 regulate stomatal development in response to light quantity. - Curr. Biol. 19: 229-234, 2009.

Casson S.A., Hetherington A.M.: Phytochrome B is required for light-mediated systemic control of stomatal development. Curr. Biol. 24: 1216-1221, 2014.

Hetherington A.M., Woodward F.I.: The role of stomata in sensing and driving environmental change. - Nature 424: 901-908, 2003.

Hoecker U.: The activities of the E3 ubiquitin ligase COP1/SPA, a key repressor in light signaling. - Curr. Opin. Plant Biol. 37: 63-69, 2017.

Hronková M., Wiesnerová D., Šimková M. et al.: Light-induced STOMAGEN-mediated stomatal development in Arabidopsis leaves. - J. Exp. Bot. 66: 4621-4630, 2015.

Hunt L., Bailey K.J., Gray J.E.: The signalling peptide EPFL9 is a positive regulator of stomatal development. - New Phytol. 186: 609-614, 2010.

Hunt L., Gray J.E.: The signaling peptide EPF2 controls asymmetric cell divisions during stomatal development. - Curr. Biol. 19: 864-869, 2009.

Jewaria P.K., Hara T., Tanaka H. et al.: Differential effects of the peptides stomagen, EPF1 and EPF2 on activation of map kinase MPK6 and the SPCH protein level. - Plant Cell Physiol. 54: 1253-1262, 2013.

Kanaoka M.M., Pillitteri L.J., Fujii H. et al.: SCREAM/ICE1 and SCREAM2 specify three cell-state transitional steps leading to Arabidopsis stomatal differentiation. - Plant Cell 20: 1775-1785, 2008.

Kang C.Y., Lian H.L., Wang F.F. et al.: Cryptochromes, phytochromes, and COP1 regulate light-controlled stomatal development in Arabidopsis. - Plant Cell 21: 2624-2641, 2009.

Kondo T., Kajita R., Miyazaki A. et al.: Stomatal density is controlled by a mesophyll-derived signaling molecule. - Plant Cell Physiol. 51: 1-8, 2010.

Lampard G.R., Bergmann D.C.: A shout-out to stomatal development: How the bHLH proteins SPEECHLESS, MUTE and FAMA regulate cell division and cell fate. - Plant Signal. Behav. 2: 290-292, 2007.

Le J., Zou J., Yang K. et al.: Signaling to stomatal initiation and cell division. - Front. Plant Sci. 5: 297, 2014.

Lee J.H., Jung J.H., Park C.M.: Light inhibits COP1-mediated degradation of ICE transcription factors to induce stomatal development in Arabidopsis. - Plant Cell 29: 2817-2830, 2017.

Lin C.: Blue light receptors and signal transduction. - Plant Cell 14: 207-225, 2002.

Liu T.D., Zhang X.W., Xu Y. et al.: Light quality modifies the expression of photosynthetic genes in maize seedlings. Photosynthetica 55: 360-367, 2017.

Mao J., Zhang Y.C., Sang Y. et al.: A role for Arabidopsis cryptochromes and COP1 in the regulation of stomatal opening. - P. Natl. Acad. Sci. USA 102: 12270-12275, 2005.

Ohashi-Ito K., Bergmann D.C.: Arabidopsis FAMA controls the final proliferation/differentiation switch during stomatal development. - Plant Cell 18: 2493-2505, 2006.

Ohki S., Takeuchi M., Mori M.: The NMR structure of stomagen reveals the basis of stomatal density regulation by plant peptide hormones. - Nat. Commun. 2: 512-519, 2011.

Pillitteri L.J., Sloan D.B., Bogenschutz N.L., Torii K.U.: Termination of asymmetric cell division and differentiation of stomata. - Nature 445: 501-505, 2007.

Sugano S.S., Shimada T., Imai Y. et al.: Stomagen positively regulates stomatal density in Arabidopsis. - Nature 463: 241-244, 2010

Torii K.U.: Mix-and-match: ligand-receptor pairs in stomatal development and beyond. - Trends Plant Sci. 17: 711-719, 2012.

Wang H., Ma L.G., Li J.M. et al.: Direct interaction of Arabidopsis cryptochromes with COP1 in light control development. Science 294: 154-158, 2001.

Yang L.Y., Wang L.T., Ma J.H. et al.: Effects of light quality on growth and development, photosynthetic characteristics and content of carbohydrates in tobacco (Nicotiana tabacum L.) plants. - Photosynthetica 55: 467-477, 2017.

Zheng L., Van Labeke M.C.: Long-term effects of red- and bluelight emitting diodes on leaf anatomy and photosynthetic efficiency of three ornamental pot plants. - Front. Plant Sci. 8: 917, 2017.

(C) The authors. This is an open access article distributed under the terms of the Creative Commons BY-NC-ND Licence. 